\title{
Isolation of Plant Photosystem II Complexes by Fractional Solubilization
}

\author{
Patrycja Haniewicz', Davide Floris², Domenica Farci'2, Joanna Kirkpatrick ${ }^{3}$, \\ Maria C. Loi ${ }^{2}$, Claudia Büchel/4, Matthias Bochtler ${ }^{1,5}$ and Dario Piano ${ }^{1,2 *}$
}

' Laboratory of Structural Biology, Department of Molecular Biology, International Institute of Molecular and Cell Biology, Warsaw, Poland, ${ }^{2}$ Laboratory of Photosynthesis and Photobiology, Department of Life and Environmental Sciences, University of Cagliari, Cagliari, Italy, ${ }^{3}$ Proteomics Core Facility, European Molecular Biology Laboratory, Heidelberg, Germany, ${ }^{4}$ Laboratory of Plant Cell Physiology, Institute of Molecular Biosciences, Goethe-University Frankfurt, Frankfurt am Main, Germany, ${ }^{5}$ Department of Bioinformatics, Institute of Biochemistry and Biophysics, Warsaw, Poland

Photosystem II (PSII) occurs in different forms and supercomplexes in thylakoid membranes. Using a transplastomic strain of Nicotiana tabacum histidine tagged on the subunit PsbE, we have previously shown that a mild extraction protocol with $\beta$-dodecylmaltoside enriches PSII characteristic of lamellae and grana margins. Here, we characterize residual granal PSII that is not extracted by this first solubilization step. Using affinity purification, we demonstrate that this PSII fraction consists of PSII-LHCII mega- and supercomplexes, PSIl dimers, and PSII monomers, which were separated by gel filtration and functionally characterized. Our findings represent an alternative demonstration of different PSII populations in thylakoid membranes, and they make it possible to prepare PSII-LHCII supercomplexes in high yield.

Keywords: photosystem II, PSII-LHCII supercomplex, PSII-LHCII megacomplex, thylakoid membranes, Nicotiana tabacum, oligomeric state

\section{INTRODUCTION}

Oxygenic photosynthesis is one of the key processes sustaining the life on our planet by providing the biosphere with oxygen and sugars. Photosystem II (PSII) is a membrane protein complex that plays an essential role in oxygenic photosynthesis. Sunlight drives the splitting of water into oxygen, electrons, and protons (Cardona et al., 2012), but it also causes photodamage, which is minimized by photoprotection in conditions of excess light and repaired by PSII turnover in all conditions. The distribution of PSII in different complexes within the membranes reflects PSII assembly and repair (Pokorska et al., 2009), as well as optimization for efficient usage of light while avoiding or limiting damage (Boekema et al., 1995; Dekker and Boekema, 2005; Takahashi et al., 2009; Watanabe et al., 2009; Pagliano et al., 2011). In lamellae and the marginal grana, PSII is assembled de novo or repaired. Exhausted PSII complexes formed in the grana cores migrate to grana margins and lamellae, while being replaced by new and fully functional complexes (Edelman and Mattoo, 2008; Nixon et al., 2010; Puthiyaveetil et al., 2014; Tomizioli et al., 2014). With respect to PSII, lamellae and grana margins may thus be considered as the "nursery", while the grana cores act as the "photochemical plant" of thylakoids.

Five main forms of PSII are thought to occur in vivo: the monomeric (PSIIm or C), the dimeric (PSIId or $\mathrm{C}_{2}$ ), the incomplete form free of the antenna component CP43 (RC-CP47) and finally the 
two PSII complexes, consisting of several combinations of $\mathrm{C}_{2}$ with the trimeric Light Harvesting Complex II (LHCII), which may bind $\mathrm{C}_{2}$ strongly $(\mathrm{S})$ or mildly $(\mathrm{M})$ via the socalled minor antenna complexes (CP24, CP26, and CP29). Their assembly leads to higher photosynthetic units called PSII-LHCII supercomplex (PSIIsc or $\mathrm{C}_{2} \mathrm{~S}_{2}$ ) and megacomplex (PSIImc or $\mathrm{C}_{2} \mathrm{~S}_{2} \mathrm{M}_{2}$; Boekema et al., 1999; Eshaghi et al., 1999; de Bianchi et al., 2008; Caffarri et al., 2009). The five PSII types are localized in different regions of the thylakoid membranes (Danielsson et al., 2006). In particular PSIIm and RC-CP47 are typically localized in the lamellae and peripheral part of the grana, being the main constituents of the "nursery" region, whereas PSIId, $\mathrm{C}_{2} \mathrm{~S}_{2}, \mathrm{C}_{2} \mathrm{~S}_{2} \mathrm{M}_{2}$ are localized in the grana cores, being the main constituents of the functional side of the thylakoid membranes.

We have recently reported the purification of an inactive form of PSIIm that contains the subunit PsbS and appears as one of the main PSII forms associated with the lamellae region of the thylakoid membranes (Haniewicz et al., 2013). In this work, we now describe the fraction that is not solubilized by the mild-extraction protocol. This fraction, that requires harsher extraction conditions, was characterized in order to reveal whether it may contain the grana complexes opening the way for their isolation and characterization. The material solubilized in the second step is characterized by Blue native polyacrylamide gel electrophoresis (BN-PAGE) and mass spectrometry (MS), which together provide information on native molecular mass and subunit composition. After solubilization, granal thylakoids were also subjected to Immobilized-Metal Affinity Chromatography (IMAC) and subsequent Size Exclusion Chromatography (SEC). In the latter step, PSII complexes, supercomplexes, and megacomplexes could be separated, further characterized and compared using several techniques. This procedure allows the chromatography isolation of preparative amounts of highly pure $\mathrm{C}, \mathrm{C}_{2}, \mathrm{C}_{2} \mathrm{~S}_{2}$ particles and represents a step further to their structural and functional characterization. The described procedure also provides a direct biochemical probe of PSII organization and distribution in thylakoid membranes.

\section{MATERIALS AND METHODS}

\section{Growth of Tobacco Plants}

Plant material was obtained from a transplastomic strain of Nicotiana tabacum that carries a hexa-histidine tag at the $5^{\prime}$ end of the gene coding for the PsbE subunit (Fey et al., 2008). The plants were grown for 10-12 weeks with a 50\% relative humidity at a constant temperature of $25^{\circ} \mathrm{C}$ and under a light regime of $12 \mathrm{~h} /$ day, with a light intensity of 150-200 $\mu \mathrm{mol}$ photons $/\left(\mathrm{s} \mathrm{m}^{2}\right)$.

\section{Thylakoid Preparation}

Thylakoid membranes were purified as reported previously by Fey et al. (2008), but in the last centrifugation step they were resuspended in $20 \mathrm{mM}$ MES-NaOH, pH 6.5; $100 \mathrm{mM} \mathrm{NaCl}$; $5 \mathrm{mM} \mathrm{MgCl}_{2} ; 10 \mathrm{mM} \mathrm{NaHCO}_{3} ; 12.5 \%$ (v/v) glycerol.

\section{Thylakoids Solubilization and PSII Core Complex Purification by Affinity Chromatography}

Thylakoid fractions were obtained routinely and with high reproducibility. Lamellar fractions were obtained by using the procedure reported in Haniewicz et al. (2013). Minimal modifications were introduced with the aim to ensure a complete removal of the peripheral grana (and lamellae) from the grana cores. Briefly, thylakoids membranes where solubilized for $30 \mathrm{~min}$ at $4^{\circ} \mathrm{C}$ and the final chlorophyll concentration was kept in a range between 2 and $3 \mathrm{mg} / \mathrm{ml}$ (SL) without major changes. After solubilization, the supernatant was separated from the unsolubilized fraction by spinning at $45000 \times g$. The unsolubilized granal fraction was homogenized and subsequently solubilized for $10 \mathrm{~min}$ at $4^{\circ} \mathrm{C}$ at a chlorophyll concentration of $1 \mathrm{mg} / \mathrm{ml}$ (SG), as reported in Fey et al. (2008) for the isolation of PSII complexes with LHC polypeptides bound. In both cases solubilization was carried out using $20 \mathrm{mM} \beta$-dodecylmaltoside $(\beta-\mathrm{DDM})$.

Photosystem II samples were prepared routinely using $\mathrm{Ni}$ affinity chromatography. PSII was isolated following the procedure reported in Piano et al. (2010) with the only difference that the washing buffer was free of glycerol and betaine (20 mM MES-NaOH, pH 6.5; $100 \mathrm{mM} \mathrm{NaCl} ; 10 \mathrm{mM} \mathrm{NaHCO}_{3}$; $15 \mathrm{mM}$ imidazole) and PSII cores were eluted using $40 \mathrm{mM}$ MES-NaOH, pH 6.5; $20 \mathrm{mM} \mathrm{NaCl} ; 5 \mathrm{mM} \mathrm{MgCl}_{2} ; 1 \mathrm{mM}$ $\mathrm{CaCl}_{2} ; 10 \mathrm{mM} \mathrm{NaHCO} 3 ; 400 \mathrm{mM}$ imidazole. The washing and the elution buffers contained $0.01 \%$ instead of $0.03 \%(\mathrm{w} / \mathrm{v})$ $\beta$-DDM.

\section{Size Exclusion Chromatography}

The eluted fractions from Ni-NTA chromatography were pooled and concentrated using Vivaspin 20 ultrafiltration membranes with $100 \mathrm{kDa}$ cutoff until a final volume of $200 \mu \mathrm{l}$. The protein sample was loaded on a home-made column of $80 \mathrm{ml}$ bed volume Superose 6 resin (GE Healthcare) with a diameter of $10 \mathrm{~mm}$ leading to highly reproducible separations of specific protein complexes. Protein separation and column pre-equilibration were performed in gel filtration buffer $(40 \mathrm{mM}$ MES-NaOH, $\mathrm{pH}$ 6.5; $20 \mathrm{mM} \mathrm{NaCl} ; 5 \mathrm{mM} \mathrm{MgCl}_{2} ; 1 \mathrm{mM} \mathrm{CaCl}_{2} ; 10 \mathrm{mM} \mathrm{NaHCO}_{3}$; $0.01 \%(w / v) \beta-D D M)$.

\section{Absorption Spectroscopy, Chlorophyll Determination, and Yield Calculation}

The protein content in thylakoids and purified complexes was calculated referring to the $\mathrm{Chl} a$ and $\mathrm{Chl} b$ concentrations from three independent measurements. The analysis was done photometrically in $80 \%(\mathrm{v} / \mathrm{v})$ acetone using a Pharmacia Biotech Ultrospec 4000 spectrophotometer and Chl concentrations were calculated according to Porra et al. (1989). Yield calculation in thylakoids and PSII complexes was expressed in $\mathrm{mg}$ or $\%$ of chlorophylls. Measurements were performed on samples from 10 independent purifications starting from different thylakoid stocks. For each of these 10 purifications was calculated the amount of thylakoids and the amounts of the specific PSII complexes isolated (C-PsbS, C, $\mathrm{C}_{2}, \mathrm{C}_{2} \mathrm{~S}_{2}$ ) expressing them in $\mathrm{mg}$ 
or \% of Chls with respect to the initial thylakoid amount. Finally, the amounts for each of the 5 classes (thylakoids, C-PsbS, C, $\mathrm{C}_{2}$, $\mathrm{C}_{2} \mathrm{~S}_{2}$ ) were averaged respect to the 10 independent purifications (Table 4). The values were expressed as means \pm standard deviations.

\section{Polyacrylamide Gel Electrophoresis}

Blue native polyacrylamide gel electrophoresis was routinely used as a reference to cross check the correct SG solubilization, the correct SEC profiles and to select the specific pools to be used for further structural and functional tests. According to Schägger and von Jagow (1991), native electrophoresis was performed using 3-12\% (w/v) continuous gradient gels. PSII complexes and thylakoid samples at $0.2 \mathrm{mg} \mathrm{Chl} / \mathrm{ml}$ were mixed with 0.25 volumes of Coomassie Blue Solution (5\% (v/v) serva Blue G, $750 \mathrm{mM}$ aminocaproic acid, 35\% (w/v) sucrose). The electrophoresis was carried out at $205 \mathrm{~V}$ for $5 \mathrm{~h}$ for PSII complexes, while for thylakoids it consisted in a run at $60 \mathrm{~V}$ for $12 \mathrm{~h}$. In both cases the run was performed at $4^{\circ} \mathrm{C}$. After the electrophoretic run, the gels were stained with Coomassie brilliant blue G250.

\section{Mass Spectrometry}

The BN-PAGE gel bands from the SG samples or from the SEC fractions were excised and analyzed. Samples were processed as described in Farci et al. (2014). Protein groups were assigned to bands, and qualitative estimates of protein abundance were based on an "index" obtained by dividing unweighted spectral counts (spectral count) by protein mass $(\mathrm{kDa})$. Proteins were either excluded (index below 0.25), or divided into groups with indices between 0.25 and 0.50 (marked with + in Table 3), 0.50 and 0.75 (marked with ++ in Table 3 ) and indices higher than 0.75 (marked with +++ in Table 3). Higher indices indicate greater abundance of the protein in the samples.

\section{Oxygen Evolution}

Oxygen evolution rates under light saturation were measured using a Clark-type electrode (Hansatech, England) at $20^{\circ} \mathrm{C}$. In the reaction mixture the samples were added to gel filtration buffer enriched with freshly prepared electron acceptors $(1 \mathrm{mM}$ 2,6-dichloro- $p$-benzoquinone and $1 \mathrm{mM}$ ferricyanide). The measurements were carried out at a Chls concentration of $100 \mu \mathrm{g} / \mathrm{ml}$ for thylakoids and $50 \mu \mathrm{g} / \mathrm{ml}$ for PSII samples. Activity was tested with three independent measurements on the same preparation and the values were expressed as means \pm standard deviations.

\section{Electron Microscopy}

Size Exclusion Chromatography fractions of different PSII complexes from three independent purifications were checked by Transmission Electron Microscopy (TEM). Samples were diluted in gel filtration buffer and applied on glow-discharged carbon
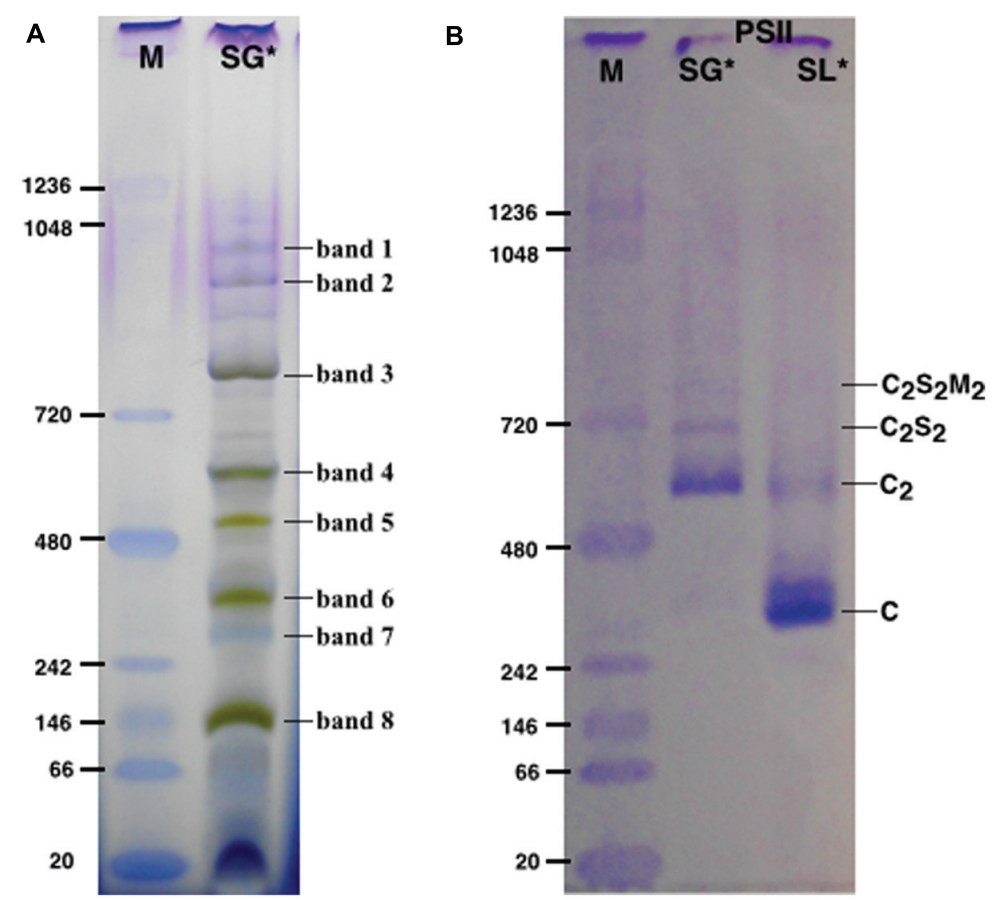

FIGURE 1 | The solubilized grana cores (SG), when resolved by Blue native polyacrylamide gel electrophoresis (BN-PAGE; A) separated into a pattern of bands equivalent to specific thylakoid complexes. The bands were attributed to a given complex on the basis of their mass spectrometry (MS) analysis (see Table 1). The PSII pools purified by NiNTA affinity chromatography are resolved by BN-PAGE (B). The lane SG is the PSII pool purified from the solubilized grana fraction, whereas lane SL represents the pool of PSIl purified from solubilized lamellae. In lanes $M$ the molecular marker ( $M$ ) was loaded. $\mathrm{C}_{2} \mathrm{~S}_{2} \mathrm{M}_{2}, \mathrm{C}_{2} \mathrm{~S}_{2}, \mathrm{C}_{2}$ and $\mathrm{C}$ are the PSII-LHCII megacomplexes, PSII-LHCII supercomplexes, PSIl dimers and PSII monomers, respectively. *The BN-PAGE used must be considered reliable in the mass range between 66 and $480 \mathrm{kDa}$ according to the molecular marker (M). Above this range, the heaviest bands appear significantly under estimated in weight. 
TABLE 1 | Mass spectrometry (MS) analysis performed on the bands of the SG samples resolved by BN-PAGE (see Figure 1A).

\begin{tabular}{|c|c|c|c|c|c|}
\hline Band & PSI (LHCI) & PSII (LHCII) & $\begin{array}{l}\text { Cyt } b_{6} f \\
\text { complex }\end{array}$ & ATP synthase & Complexes \\
\hline 1 & $\begin{array}{l}\text { PsaB, D, E, F, K } \\
\text { CAB4, 7, } 40\end{array}$ & $\begin{array}{l}\text { PsbA, B, C, D, E, O, Q } \\
\text { CP29 }\end{array}$ & - & - & $\begin{array}{l}\text { PSI and PSII } \\
\text { higher complexes }\end{array}$ \\
\hline 2 & $\begin{array}{l}\text { PsaA, B, D, F, K } \\
\text { CAB40 }\end{array}$ & PsbA, B, C, D, Q & - & - & \\
\hline 3 & $\begin{array}{l}\text { PsaA, B, D, F, L,H,K } \\
\text { CAB4,16, 21, 40, Ihca }\end{array}$ & $\begin{array}{l}\text { PsbA, B, C, D, E, O } \\
\text { CAB7, 36, 40, CP24, 26, } 29\end{array}$ & - & atpA, B & $\begin{array}{l}\text { PSIl megacomplex } \\
\left(\mathrm{C}_{2} \mathrm{~S}_{2} \mathrm{M}_{2}\right), \text { PSI-LHCII } \\
\text { ATPase }\end{array}$ \\
\hline 4 & $\begin{array}{l}\text { PsaA, B, D, E, F, L, } \\
\text { CAB4, 25, Ihca }\end{array}$ & $\begin{array}{l}\text { PsbA, B, C, D, E, O, S } \\
\text { CAB7, 13, 36, 40, CP29, Ihcb }\end{array}$ & - & $\begin{array}{l}\operatorname{atpA}, B, C, E, F \\
\text { atp } \alpha, \gamma\end{array}$ & $\begin{array}{l}\text { PSIl supercomplex } \\
\left(\mathrm{C}_{2} \mathrm{~S}_{2}\right) \\
\text { PSI-LHCII } \\
\text { ATPase }\end{array}$ \\
\hline 5 & $\begin{array}{l}\text { PsaB } \\
\text { CAB16, 21, } 40\end{array}$ & $\begin{array}{l}\text { PsbA, B, C, D, E, O, S } \\
\text { CAB7, 13, 36, 50, CP26, } 29\end{array}$ & - & - & $\begin{array}{l}\text { PSIl dimer }\left(\mathrm{C}_{2}\right) \\
\mathrm{PSI}\end{array}$ \\
\hline 6 & CAB21, 40 & $\begin{array}{l}\text { PsbA, B, C, D, E, O, Q } \\
\text { CAB7, 16, 36, 50, CP26, } 29\end{array}$ & $\begin{array}{l}\text { Cyt. f subunit } \\
\text { subunit IV }\end{array}$ & - & $\begin{array}{l}\text { PSIl monomer (C), } \\
\text { cytb }_{6} \text { f complex }\end{array}$ \\
\hline 7 & CAB40 & $\begin{array}{l}\text { PsbA, B, C, D } \\
\text { CAB7, 36, CP24, } 26\end{array}$ & - & - & $\begin{array}{l}\text { PSIl incomplete } \\
\text { monomer }\end{array}$ \\
\hline 8 & CAB21, 40 & $\begin{array}{l}\text { PsbC, D, O, P, Q, S } \\
\text { CAB7, 36, 50, CP24, 26, } 29\end{array}$ & Cyt. f subunit & $\operatorname{atp} \beta$ & Free subunits \\
\hline
\end{tabular}

For a more detailed analysis see Supplementary Table S1.

coated copper grids (400 mesh) followed by negative staining using filtered 2\% uranyl acetate. Electron microscopy was performed in a CM12 electron microscope (Philips, Eindhoven, Netherlands) operated at $80 \mathrm{kV}$. Images were recorded under low dose conditions (total dose $\sim 25 e-/ \AA^{2}$ ) with a ES500W camera (Gatan, Pleasanton, CA, USA) at a magnification of $110 \mathrm{kx}$.

\section{RESULTS}

\section{Composition of SL and SG Fractions}

In order to characterize PSII species associated to granal thylakoids, we solubilized membranes in two steps. In the first step, we used a previously described mild and long extraction procedure that selectively solubilizes only the peripheral part of the thylakoids (lamellae and grana margins; SL) leading to the chromatography isolation of monomeric PSII that binds the subunit PsbS (see protocol B samples in Table 1 of Haniewicz et al., 2013). The fraction of thylakoids not solubilized during the preparation of SL was pelleted by centrifugation, resuspended and finally subjected to a second solubilization, leading to samples with a PSII content representative for grana cores (SG) that were then subjected to BN-PAGE.
SG samples migrated on BN-PAGE resolving in bands with apparent masses from 1050 to $60 \mathrm{kDa}$ (Figure 1A). The content in thylakoid complexes of each band was identified by MS. According to this analysis, PSII was mainly present in the $\mathrm{C}_{2} \mathrm{~S}_{2} \mathrm{M}_{2}, \mathrm{C}_{2} \mathrm{~S}_{2}, \mathrm{C}_{2}$ and $\mathrm{C}$ forms (Table 1, Supplementary Table $\mathrm{S} 1)$. When incubated on ice for more than $6 \mathrm{~h}$, the SL samples, but not the SG, were characterized by a tendency to precipitate, indicating an insufficient solubilization, which led to difficulties in their characterization by BN-PAGE (data not shown). Taken together, these data suggested large differences in the properties of lamellae and grana cores of the thylakoid membranes.

\section{Oxygen Evolving Activity of SL and SG Fractions}

The oxygen evolution capacity of SL and SG samples was tested. The SL samples had a minimal activity of $19 \mu \mathrm{mol} \mathrm{O}_{2} / \mathrm{mg}$ Chl h, while the SG samples evolved 10-fold more oxygen (186 $\mu \mathrm{molO}_{2} / \mathrm{mg} \mathrm{Chl} \mathrm{h),} \mathrm{suggesting} \mathrm{the} \mathrm{presence} \mathrm{of} \mathrm{abundant}$ and functional PSII (Table 2). These findings are consistent with the accepted view of the lamellae as the assembly and/or repair region of thylakoids in which the PSII particles are mainly inactive, and of the grana in which PSII is in an optimal chemicalphysical environment that keeps it very active (Aro et al., 2005).

TABLE 2 | Rates of oxygen evolution of solubilized lamellar (SL) thylakoids, solubilized granal (SG) thylakoids and of PSII-LHCII megacomplexes (C2S2M2), PSII-LHCII supercomplexes $\left(\mathrm{C}_{2} \mathrm{~S}_{2}\right)$, PSII dimers (C2), and PSII monomers (C).

\begin{tabular}{|c|c|c|c|c|c|}
\hline \multicolumn{2}{|c|}{ Thylakoids* } & \multicolumn{4}{|c|}{ PSII purified samples* } \\
\hline SL & SG & PSII monomers (C) & PSIl dimers $\left(\mathbf{C}_{2}\right)$ & PSII-LHCII supercomplexes $\left(\mathrm{C}_{2} \mathrm{~S}_{2}\right)$ & PSII-LHCII megacomplexes $\left(\mathrm{C}_{2} \mathrm{~S}_{2} \mathrm{M}_{2}\right)$ \\
\hline $19 \pm 2$ & $186 \pm 5$ & $960 \pm 5$ & $1360 \pm 12$ & $1030 \pm 10$ & not determined \\
\hline
\end{tabular}




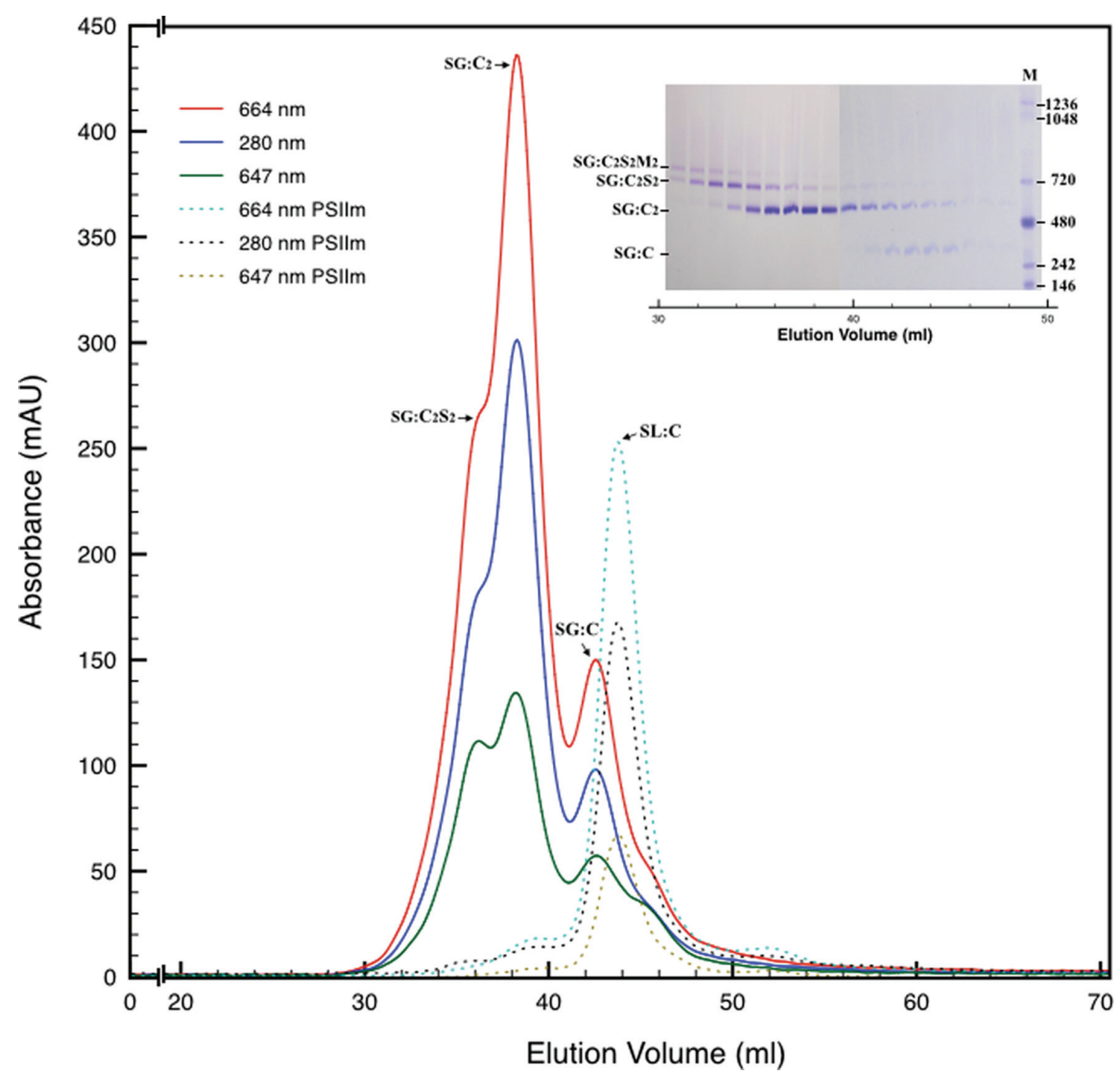

FIGURE 2 | Size exclusion chromatography of the PSII pool isolated from SG thylakoids by affinity chromatography (solid lines). All the measurements were recorded at three different wavelengths: $280 \mathrm{~nm}$ (proteins), $664 \mathrm{~nm}$ (chlorophyll a), $647 \mathrm{~nm}$ (chlorophyll $b$ ). In the inset are shown the elution fractions analyzed by BN-PAGE, confirming the partial separation of several oligomeric states of PSII. M is the molecular marker. The mostly monomeric PSIl pool isolated from SL thylakoids by affinity chromatography (dotted lines) was used as a mass reference. In the chromatograms and in the inset SG: $\mathrm{C}_{2} \mathrm{~S}_{2} \mathrm{M}_{2}, \mathrm{SG}_{\mathrm{i}} \mathrm{C}_{2} \mathrm{~S}_{2}, \mathrm{SG}_{\mathrm{C}} \mathrm{C}_{2}$, and $\mathrm{SG}: \mathrm{C}$ are the PSII-LHCII megacomplexes, PSII-LHCII supercomplexes, PSIl dimers, and PSII monomers of grana origin (SG), respectively; SL:C are PSII monomer from lamellae (SL).

\section{Oligomeric States of PSII Complexes from the SG Fraction}

The SG and SL samples were subjected to further PSII separation steps after solubilization. The histidine tag on PsbE of the transplastomic plants made it possible to purify PSII by $\mathrm{Ni}$ NTA (Fey et al., 2008). After purification, the oligomeric profile of the obtained SG-PSII was assessed by BN-PAGE and the composition compared with the already characterized SL-PSII (Haniewicz et al., 2013), showing a significant difference between the oligomeric patterns of the two PSII samples (Figure 1B). As clearly shown in Figure 1B, mainly $\mathrm{C}$ and sometimes incomplete PSII forms such as RC-CP47 can be obtained from SL, while SG is a mixture of $\mathrm{C}_{2}$ and $\mathrm{C}_{2} \mathrm{~S}_{2}$ with the frequent presence of small $\mathrm{C}_{2} \mathrm{~S}_{2} \mathrm{M}_{2}$ amounts.

\section{The Different SG-PSII Species can be Separated by Size Exclusion Chromatography}

Next, we attempted to separate the different PSII components obtained by the Ni-NTA chromatography from the SG-PSII samples by means of SEC. SG-PSII samples resolved into a characteristic and reproducible profile consisting of a shoulder and two peaks (Figure 2). In contrast, the same procedure for the SL-PSII samples led to a single peak corresponding to monomeric PSII confirming the BN-PAGE analysis on the NiNTA pool (Figures $\mathbf{2}$ and $\mathbf{1 B}$ ) and previous results (Haniewicz et al., 2013). The collected fractions from the SEC analysis on the SG-PSII samples were checked by BN-PAGE and thus divided into four pools corresponding to samples enriched in 
TABLE 3 | Mass spectrometry analysis performed on the bands of the SEC fractions resolved by BN-PAGE.

\begin{tabular}{|c|c|c|c|c|c|c|c|c|c|c|c|}
\hline & \multirow[t]{2}{*}{ Name } & \multirow[t]{2}{*}{ Accession Number } & \multirow[t]{2}{*}{$\begin{array}{l}\text { Mass } \\
(\mathrm{kDa})\end{array}$} & \multicolumn{4}{|c|}{$\begin{array}{l}\text { Qualitative (unweighted } \\
\text { subunit presence) }\end{array}$} & \multicolumn{4}{|c|}{$\begin{array}{l}\text { Quantitative* (weighted subunit } \\
\text { presence) }\end{array}$} \\
\hline & & & & C & $\mathrm{c}_{2}$ & $\mathrm{c}_{2} \mathrm{~s}_{2}$ & $\mathrm{C}_{2} \mathrm{~S}_{2} \mathrm{M}_{2}$ & C & $\mathrm{C}_{2}$ & $\mathrm{C}_{2} \mathrm{~s}_{2}$ & $\mathrm{C}_{2} \mathrm{~S}_{2} \mathrm{M}_{2}$ \\
\hline \multirow{13}{*}{ 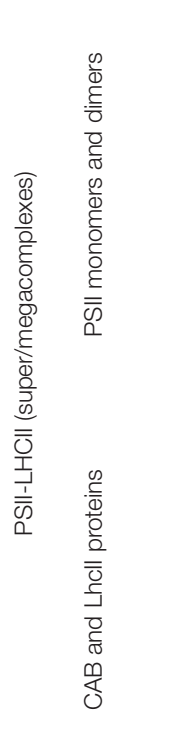 } & PSB_A (D1) & PSBA_TOBAC & 39 & + & + & + & + & +++ & +++ & +++ & +++ \\
\hline & PSB_B (CP47) & PSBB_TOBAC & 56 & + & + & + & + & +++ & +++ & +++ & +++ \\
\hline & PCB_C (CP43) & PSBC_TOBAC & 52 & + & + & + & + & +++ & +++ & +++ & +++ \\
\hline & PSB_H & PSBH_TOBAC & 8 & + & + & + & + & +++ & +++ & +++ & +++ \\
\hline & PSB_L & PBL_TOBAC & 4 & - & + & + & - & - & +++ & +++ & - \\
\hline & PSB_O (33kDa) & Q84QE8 & 35 & + & + & + & + & - & ++ & +++ & + \\
\hline & PSB_O (33kDa) & PSBO_TOBAC & 35 & - & + & + & + & - & ++ & +++ & + \\
\hline & PSB_R & PSBR_TOBAC & 14 & - & + & + & + & - & + & + & - \\
\hline & Lhcb2 (CB23) & CB23_TOBAC (+1) & 29 & - & + & + & + & - & - & +++ & ++ \\
\hline & Lhcb3 & A0A076L1Y1_TOBAC $(+1)$ & 29 & - & - & + & + & - & - & - & - \\
\hline & Lhcb4 (CP29) & QOPWS7_TOBAC & 31 & + & + & + & + & - & - & +++ & +++ \\
\hline & Lhcb5 (CP26) & QOPWS5_TOBAC & 30 & - & + & + & + & - & - & +++ & +++ \\
\hline & Lhcb6 (CP24) & QOPWS6_TOBAC & 27 & - & + & + & + & - & - & + & + \\
\hline \multirow{2}{*}{$\begin{array}{l}\text { Plastidial ATP } \\
\text { synthase }\end{array}$} & ATP_A ( $\alpha$-subunit) & ATPA_TOBAC & 55 & - & + & - & - & - & - & - & - \\
\hline & ATP_B ( $\beta$-subunit) & ATPB_TOBAC (+1) & 54 & - & + & - & - & - & - & - & - \\
\hline- & $\begin{array}{l}37 \mathrm{kDa} \text { inner } \\
\text { membrane } \\
\text { polypeptide }\end{array}$ & Q40501_TOBAC & 38 & - & + & + & + & - & - & - & - \\
\hline
\end{tabular}

In the table are shown the protein composition and the relative genes for each of the PSII types separated (Figure 2 inset). $C_{2} S_{2} M_{2}, C_{2} S_{2}, C_{2}, C$ are the PSII-LHCII megacomplexes, PSII-LHCII supercomplexes, PSIl dimers, and PSII monomers, respectively. ${ }^{*}$ Unweighted spectrum count/MASS $(\mathrm{kDa})=i ;+=0.25 \leq i \leq 0.50$; $++=0.50 \leq i \leq 0.75 ;+++=i>0.75$ (for details see Materials and Methods).

a specific complex primarily identified by its apparent size on BN-PAGE (Figure 2 inset). The identity of each complex was finally confirmed by MS analysis (Table 3, Supplementary Table S2). Furthermore, the $\mathrm{C}_{2} \mathrm{~S}_{2}$ and $\mathrm{C}_{2}$ complexes were also analyzed by TEM (Figure 3), demonstrating the sizes reported for $\mathrm{C}_{2}$ and $\mathrm{C}_{2} \mathrm{~S}_{2}$ (Aro et al., 2005; Nield and Barber, 2006), the latter most easily discernible from the side views (circled in Figure 3A). Unfortunately, $\mathrm{C}_{2} \mathrm{~S}_{2} \mathrm{M}_{2}$ complexes could not be analyzed by TEM, because they were obtained in low amounts and eluted at the beginning of the $\mathrm{C}_{2} \mathrm{~S}_{2}$ peak in SEC.

\section{The Isolated PSII Types Evolve Oxygen at Very Different Rates}

The different PSII complexes obtained by SEC were tested for their oxygen evolving capacity. The pool of $\mathrm{C}_{2} \mathrm{~S}_{2} \mathrm{M}_{2}$ was excluded from this analysis since, as mentioned above, it was not perfectly separated from the $\mathrm{C}_{2} \mathrm{~S}_{2}$. Each one of the others pools, represented by a dominant PSII type, were found to be fully functional and characterized by oxygen evolution rates comparable with other reports in literature (Kihara et al., 2014; Table 2). As oxygen evolution per Chl is reported, and because $\mathrm{C}_{2} \mathrm{~S}_{2}$ binds about three times more Chls per reaction center than cores (around 210/reaction center as opposed to around 70; Ferreira et al., 2004; Liu et al., 2004; Loll et al., 2005; Barros et al., 2009), the $C_{2} S_{2}$ complexes are characterized by the highest activity.

\section{Mass Spectrometry Analysis on the Four PSII Types Showed Significant Differences in Subunit Composition}

The BN-PAGE bands obtained from the SEC fractions (Figure 2 inset) were excised and directly analyzed by MS to characterize subunit content (Table 3, Supplementary Table S2). The main core subunits D1, D2, CP43, CP47, PsbE, PsbH, PsbL, PsbO, and PsbR were found in this analysis. The subunit PsbL, an important dimerization factor (Suorsa et al., 2004), was absent from the $\mathrm{C}$ and $\mathrm{C}_{2} \mathrm{~S}_{2} \mathrm{M}_{2}$ types. PsbR was identified only in the $\mathrm{C}_{2}$ and $\mathrm{C}_{2} \mathrm{~S}_{2}$ types, while PsbF was not found. The partial or complete 
absence of these three small peptides is most likely due to their small masses, which limit their successful MS identification (Shi et al., 2012). The PsbO subunit was found in both isoforms. The $\mathrm{C}_{2}$ and $\mathrm{C}$ forms appeared to contain lower amounts of this protein, even though they showed a fair water splitting capacity (Table 2). PsbP and PsbQ, the other main water splitting subunits, were not identified in the analyzed complexes. Specific subunits were characteristic for a given oligomeric state. As expected, it was found that the subunits CP24, CP26, and CP29 were present just in traces or absent in the $\mathrm{C}$ and $\mathrm{C}_{2}$, while they were found to be characteristic for $\mathrm{C}_{2} \mathrm{~S}_{2}$ and $\mathrm{C}_{2} \mathrm{~S}_{2} \mathrm{M}_{2}$. Also the proteins Lhcb1, Lhcb2 and Lhcb3 were found associated with the $\mathrm{C}_{2} \mathrm{~S}_{2}$ and $\mathrm{C}_{2} \mathrm{~S}_{2} \mathrm{M}_{2}$ complexes. In particular Lhcb3 was found to be present only in traces and Lhcb1 was present in four different isoforms. One of these, CB25, was specific for the $\mathrm{C}_{2} \mathrm{~S}_{2}$ form.

\section{DISCUSSION}

\section{The Oxygen Evolving Properties Reflect the PSII Cycle}

The distribution of PSII in thylakoid membranes reflects the PSII cycle (Tomizioli et al., 2014). Grana cores sustain water splitting at the highest rates. Lamellae and grana margins are mainly required to recycle and replace PSII, which at these stages is still exposed to light, but requires protection. In agreement with this general picture, PSII isolated from SL samples is inactive, monomeric and retains PsbS. The presence of PsbS and low PSII activity in SL samples fit with the low activity of lamellar PSII reported earlier and with the role of PsbS in nonphotochemical quenching (Allen, 2002; Munekage et al., 2002; Gerotto et al., 2015). PsbS may keep PSII in an inactive and protected state for assembly or repair. PSII from the SG samples is a mix of four different types that are very active in oxygen evolution, also in agreement with the current model for the PSII cycle.

\section{PsbP and PsbQ}

All active PSII complexes from grana lacked the PsbP and PsbQ subunits, which were found earlier in the SL fractions (Haniewicz et al., 2013). As PsbP and PsbQ are thought to enhance the PSII activity and the Oxygen Evolving Complex (OEC) stability (Bricker and Frankel, 2011), this finding is

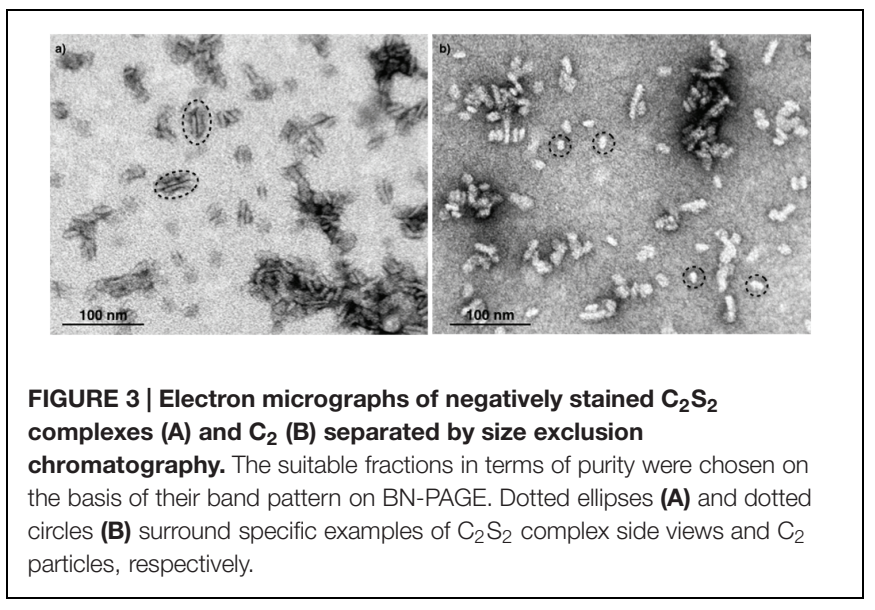

surprising, but it is not unprecedented. PsbP and PsbQ were also absent after a very mild membrane solubilization in a study on D1 subunit processing (Figure 5B from Che et al., 2013). However, the two subunits were present in several earlier PSII preparations, at least judging from SDS-PAGE gel migration patterns (Boekema et al., 2000; Nield et al., 2000; Caffarri et al., 2009). Mummadisetti et al. (2014) also detected PsbP and PsbQ by Western blotting, but, unfortunately, they did not determine the oligomeric PSII state by BN-PAGE and/or SEC. Boekema et al. (1998) detected PsbP in PSIILHCII supercomplexes, but other studies did not corroborate this finding (Caffarri et al., 2009; Barera et al., 2012). Thus, PsbP and PsbQ may only be present in some assembly stages of PSII complexes or, more likely, in the very active forms of PSII they may be loosely bound due to their high sensitivity to damage.

\section{Lhcb3 and CP24}

Low levels of Lhcb3 and CP24 were detected for both $\mathrm{C}_{2} \mathrm{~S}_{2} \mathrm{M}_{2}$ and $\mathrm{C}_{2} \mathrm{~S}_{2}$ complexes. The latter has not been reported to contain these two subunits. However, special hemi-types, intermediate between the $\mathrm{C}_{2} \mathrm{~S}_{2}$ and the $\mathrm{C}_{2} \mathrm{~S}_{2} \mathrm{M}_{2}$ types, which retain both extra subunits, have been described and could fit well with the copresence in our $\mathrm{C}_{2} \mathrm{~S}_{2}$ samples. In Caffarri et al. (2009) is reported a so-called $\mathrm{C}_{2} \mathrm{SM}$ complex containing only one $\mathrm{S}$ trimer and one $\mathrm{M}$ trimer that are bridged through CP29, CP24, and CP26 to the dimeric core. This complex has almost the same mass $(1040 \mathrm{kDa})$ as the $\mathrm{C}_{2} \mathrm{~S}_{2}$ complex $(1100 \mathrm{kDa})$, and therefore co-migrates in

TABLE 4 | Purification yields for the isolated PSII types.

\begin{tabular}{|c|c|c|c|c|c|c|}
\hline & \multirow[t]{3}{*}{ Thylakoid membranes } & \multicolumn{5}{|c|}{ Purification } \\
\hline & & \multirow{2}{*}{$\begin{array}{c}\text { Mild solubilization (SL) } \\
\text { C-PsbS }\end{array}$} & \multicolumn{4}{|c|}{ Harsh solubilization (SG) } \\
\hline & & & C & $\mathrm{C}_{2}$ & $\mathrm{C}_{2} \mathrm{~S}_{2}$ & $\mathrm{C}_{2} \mathrm{~S}_{2} \mathrm{M}_{2}$ \\
\hline Mass (mg) & $34.97 \pm 3.8$ & $0.95 \pm 0.11$ & $0.19 \pm 0.01$ & $9.55 \pm 0.81$ & $1.91 \pm 0.18$ & Not determined \\
\hline Yield (\%) & 100 & $2.73 \pm 0.31$ & $0.55 \pm 0.04$ & $27.30 \pm 2.33$ & $5.45 \pm 0.51$ & Not determined \\
\hline
\end{tabular}


BN-PAGE (and also co-sediments in a sucrose gradient; Caffarri et al., 2009). Another hemi-type, called $\mathrm{C}_{2} \mathrm{~S}_{2}{ }^{+}$, is reported to have similar characteristics (Pietrzykowska et al., 2014). It is likely that these incomplete forms are assembly intermediates on the way to $\mathrm{C}_{2} \mathrm{~S}_{2} \mathrm{M}_{2}$, or result from its disintegration, either in membranes or during solubilization or purification.

\section{Isolation of Supercomplexes by Coupling Differential Solubilization and Chromatography}

The organization of the thylakoids and in particular the distribution of pigment-protein complexes within are very well characterized (Danielsson et al., 2006). This and other works all demonstrate substantial heterogeneity of the PSII species in the thylakoid membranes (Boekema et al., 1995; Dekker and Boekema, 2005; Danielsson et al., 2006; Takahashi et al., 2009; Watanabe et al., 2009; Pagliano et al., 2011). Here, we present a differential solubilization of the thylakoid membranes, which leads to the isolation of samples with different oligomeric patterns of PSII and other thylakoid complexes (Figure 1A). Conceptually, our method to access to the grana cores fraction, in which is found highly active PSII, is similar to the method described by Berthold et al. (1981), the so-called BBY preparation, which also removes lamellae and peripheral granal regions. In contrast to the BBY method, the new method has the advantage to avoid the breakdown of granal PSII core complexes ( $\mathrm{C}$ and $\mathrm{RC}-\mathrm{CP} 47$ are found at most in small quantities).

\section{CONCLUSION}

It has to be remarked that the mild nature of the solubilization protocol permits a full separation between PSII monomers and dimers, and makes it possible to prepare $\mathrm{C}_{2} \mathrm{~S}_{2}$ in larger quantities without density gradients on sucrose or Percoll (Ghanotakis et al., 1987; Caffarri et al., 2004), (Table 4).

\section{REFERENCES}

Allen, J. (2002). Photosynthesis of ATP-electrons, proton pumps, rotors, and poise. Cell 110, 273-276.

Aro, E. M., Suorsa, M., Rokka, A., Allahverdiyeva, Y., Paakkarinen, V., Saleem, A. et al. (2005). Dynamics of photosystem II: a proteomic approach to thylakoid protein complexes. J. Exp. Bot. 56, 347-356. doi: 10.1093/jxb/eri041

Barera, S., Pagliano, C., Pape, T., Saracco, G., and Barber, J. (2012). Characterization of PSII-LHCII supercomplexes isolated from pea thylakoid membrane by onestep treatment with $\alpha$ - and $\beta$-dodecyl-D-maltoside. Philos. Trans. R. Soc. Lond. B Biol. Sci. 367, 3389-3399. doi: 10.1098/rstb.2012.0056

Barros, T., Royant, A., Standfuss, J., Dreuw, A., and Kühlbrandt, W. (2009). Crystal structure of plant light-harvesting complex shows the active, energy-transmitting state. EMBO J. 28, 298-306. doi: 10.1038/emboj.20 08.276

Berthold, A. D., Babcock, G. T., and Yocum, C. (1981). A highly resolved, oxygen evolving photosystem II preparation from spinach thylakoid membranes. FEBS Lett. 134, 231-234. doi: 10.1016/0014-5793(81) 80608-4

Boekema, E. J., Hankamer, B., Bald, D., Kruip, J., Nield, J., Boonstra, A. F., et al. (1995). Supramolecular structure of the photosystem II complex from

\section{AUTHOR CONTRIBUTIONS}

DP conceived the study, participated in its design and coordination, carried out the membranes preparation, participated in the biochemical studies, and drafted the manuscript. $\mathrm{PH}$ participated in the design of the study, participated the biochemical studies, and participated in the membranes preparation. D. Floris carried out the membranes preparation, participated in the biochemical studies. D. Farci carried out the membranes preparation, participated in the biochemical studies. JK carried out the MS analysis. ML participated in the preparation of membranes. $\mathrm{CB}$ contributed to the electron microscopy analysis and helped to draft the manuscript. MB participated in the design of the study and helped to draft the manuscript.

\section{ACKNOWLEDGMENTS}

This work was carried out with the support of the program Homing Plus (Foundation for Polish Science) grant No: 2012-6/10 co-financed by the European Union under the European Regional Development Funds; the program PRELUDIUM (National Science Centre) grant number DEC-2012/05/05/N/NZ1/01922; the Marie Curie program "European Reintegration Grant" (PERG05-GA2009-247789); the program "FSE SARDEGNA 20072013, Legge Regionale 7 agosto 2007, n. 7, Promozione della ricerca scientifica e dell'innovazione tecnologica in Sardegna”.

\section{SUPPLEMENTARY MATERIAL}

The Supplementary Material for this article can be found online at: http://journal.frontiersin.org/article/10.3389/fpls.2015.01100

green plants and cyanobacteria. Proc. Natl. Acad. Sci. U.S.A. 92, 175-179. doi: 10.1073/pnas.92.1.175

Boekema, E. J., Nield, J., Hankamer, B., and Barber, J. (1998). Localization of the $23-\mathrm{kDa}$ subunit of the oxygen-evolving complex of photosystem II by electron microscopy. Eur. J. Biochem. 252, 268-276. doi: 10.1046/j.14321327.1998.2520268.x

Boekema, E. J., van Breemen, J. F. L., van Roon, H., and Dekker, J. P. (2000). Conformational changes in photosystem II supercomplexes upon removal of extrinsic subunits. Biochemistry 39, 12907-12915. doi: 10.1021/bi0009183

Boekema, E. J., Van Roon, H., Van Breemen, J. F., and Dekker, J. P. (1999). Supramolecular organization of photosystem II and its light-harvesting antenna in partially solubilized photosystem II membranes. Eur. J. Biochem. 266, 444-452. doi: 10.1046/j.1432-1327.1999.00876.x

Bricker, T. M., and Frankel, L. K. (2011). Auxiliary functions of the PsbO, PsbP and PsbQ proteins of higher plant Photosystem II: a critical analysis. J. Photochem. Photobiol. B Biol. 104, 165-178. doi: 10.1016/j.jphotobiol.2011. 01.025

Caffarri, S., Croce, R., Cattivelli, L., and Bassi, R. (2004). A look within LHCII: differential analysis of the Lhcb1-3 complexes building the major trimeric antenna complex of higher-plant photosynthesis. Biochemistry 43, 9467-9476. doi: $10.1021 / \mathrm{bi036265i}$ 
Caffarri, S., Kouril, R., Kereïche, S., Boekema, E. J., and Croce, R. (2009). Functional architecture of higher plant photosystem II supercomplexes. EMBO J. 28, 3052-3063. doi: 10.1038/emboj.2009.232

Cardona, T., Sedoud, A., Cox, N., and Rutherford, A. W. (2012). Charge separation in photosystem II: a comparative and evolutionary overview. Biochim. Biophys. Acta 1817, 26-43. doi: 10.1016/j.bbabio.2011.07.012

Che, Y., Fu, A., Hou, X., McDonald, K., Buchanan, B. B., Huang, W., et al. (2013). C-terminal processing of reaction center protein D1 is essential for the function and assembly of photosystem II in Arabidopsis. Proc. Natl. Acad. Sci. U.S.A. 110, 16247-16252. doi: 10.1073/pnas.1313894110

Danielsson, R., Suorsa, M., Paakkarinen, V., Albertsson, P., Styring, S., Aro, E. M., et al. (2006). Dimeric and monomeric organization of photosystem II. J. Biol. Chem. 281, 14241-14249. doi: 10.1074/jbc.M600634200

de Bianchi, S., Dall'Osto, L., Tognon, G., Morosinotto, T., and Bassi, R. (2008). Minor antenna proteins CP24 and CP26 affect the interactions between photosystem II subunits and the electron transport rate in grana membranes of Arabidopsis. Plant Cell 20, 1012-1028. doi: 10.1105/tpc.107.055749

Dekker, J. P., and Boekema, E. J. (2005). Supramolecular organization of thylakoid membrane proteins in green plants. Biochim. Biophys. Acta 1706, 12-39. doi: 10.1016/j.bbabio.2004.09.009

Edelman, M., and Mattoo, A. K. (2008). D1-protein dynamic in photosystem II: the lingering enigma. Photosynth. Res. 98, 609-620. doi: 10.1007/s11120-00 8-9342-x

Eshaghi, S., Andersson, B., and Barber, J. (1999). Isolation of a highly active PSII-LHCII supercomplex from thylakoid membranes by a direct method. FEBS Lett. 446, 23-26. doi: 10.1016/S0014-5793(99) 00149-0

Farci, D., Bowler, M. W., Kirkpatrick, J., McSweeney, S., Tramontano, E., and Piano, D. (2014). New features of the cell wall of the radio-resistant bacterium Deinococcus radiodurans. Biochim. Biophys. Acta 1838, 1978-1984. doi: 10.1016/j.bbamem.2014.02.014

Ferreira, K. N., Inverson, T. M., Maghlaoui, K., Barber, J., and Iwata, S. (2004). Architecture of the photosynthetic oxygen-evolving center. Science 303, 1831-1838. doi: 10.1126/science. 1093087

Fey, H., Piano, D., Horn, R., Fischer, D., Schröder, W. P., Bock, R., et al. (2008). Isolation of highly active photosystem II core complexes with a His-tagged Cyt b559 subunit from transplastomic tobacco plants. Biochim. Biophys. Acta 1777, 1501-1509. doi: 10.1016/j.bbabio.2008.09.012

Gerotto, C., Franchin, C., Arrigoni, G., and Morosinotto, T. (2015). In vivo identification of Photosystem II Light Harvesting Complexes interacting with Photosystem II Subunit S. Plant Physiol. 168, 1747-1761. doi: 10.1104/pp.15.00361

Ghanotakis, D. F., Demetriou, D. M., and Yocum, C. F. (1987). Isolation and characterization of an oxygen-evolving photosystem II reaction center core preparation and a $28 \mathrm{kDa} \mathrm{Chl}$ a binding protein. Biochim. Biophys. Acta 891, 15-21. doi: 10.1016/0005-2728(87)90078-8

Haniewicz, P., De Sanctis, D., Büchel, C., Schröder, W. P., Loi, M. C., Kieselbach, T., et al. (2013). Isolation of monomeric photosystem II that retains the subunit PsbS. Photosynth. Res. 118, 199-207. doi: 10.1007/s11120-01 3-9914-2

Kihara, S., Hartzler, D. A., and Savikhin, S. (2014). Oxygen concentration inside a functioning photosynthetic cell. Biophys. J. 106, 1882-1889. doi: 10.1016/j.bpj.2014.03.031

Liu, Z., Yan, H., Wang, K., Kuang, T., Zhang, J., Gui, L., et al. (2004). Crystal structure of spinach major light-harvesting complex at $2.72 \AA$ resolution. Nature 428, 287-292. doi: 10.1038/nature02373

Loll, B., Kern, J., Saenger, W., Zouni, A., and Biesiadka, J. (2005). Towards complete cofactor arrangement in the $3.0 \AA$ resolution structure of photosystem II. Nature 438, 1040-1044. doi: 10.1038/nature04224

Mummadisetti, M. P., Frankela, L. K., Bellamyb, H. D., Sallansc, L., Goettertb, J. S., Brylinskia, M., et al. (2014). Use of protein cross-linking and radiolytic footprinting to elucidate PsbP and PsbQ interactions within higher plant Photosystem II. Proc. Natl. Acad. Sci. U.S.A. 111, 16178-16183. doi: 10.1073/pnas.1415165111

Munekage, Y., Hojo, M., Meurer, J., Endo, T., Tasaka, M., and Shikanai, T. (2002). PGR5 is involved in cyclic electron flow around photosystem I and II essential for photoprotection in Arabidopsis. Cell 110, 361-371. doi: 10.1016/S00928674(02)00867-X
Nield, J., and Barber, J. (2006). Refinement of the structural model for the Photosystem II supercomplex of higher plants. Biochim. Biophys. Acta 1757, 353-361. doi: 10.1016/j.bbabio.2006.03.019

Nield, J., Orlova, E. V., Morris, E. P., Gowen, B., van Heel, M., and Barber, J. (2000). 3D map of the plant photosystem II supercomplex obtained by cryoelectron microscopy and single particle analysis. Nat. Struct. Biol. 7, 44-47. doi: $10.1038 / 71242$

Nixon, P. J., Michoux, F., Yu, J., Boehm, M., and Komenda, J. (2010). Recent advances in understanding the assembly and repair of photosystem II. Ann. Bot. 106, 1-16. doi: 10.1093/aob/mcq059

Pagliano, C., Chimirri, F., Saracco, G., Marsano, F., and Barber, J. (2011). Onestep isolation and biochemical characterization of highly active plant PSII monomeric core. Photosynth. Res. 108, 33-46. doi: 10.1007/s11120-011-9650-4

Piano, D., El Alaoui, S., Korza, H. J., Filipek, R., Sabala, I., Haniewicz, P., et al. (2010). Crystallization of the photosystem II core complex and its chlorophyll binding subunit CP43 from transplastomic plants of Nicotiana tabacum. Photosynth. Res. 106, 221-226. doi: 10.1007/s11120-010-9597-x

Pietrzykowska, M., Suorsa, M., Semchonok, D. A., Tikkanen, M., Boekema, E. J., Aro, E. M., et al. (2014). The light-harvesting chlorophyll a/b binding proteins Lhcb1 and Lhcb2 play complementary roles during state transitions in Arabidopsis. Plant Cell 26, 3646-3660. doi: 10.1105/tpc.114.12 7373

Pokorska, B., Zienkiewicz, M., Powikrowska, M., Drozak, A., and Romanowska, E. (2009). Differential turnover of the photosystem II reaction centre D1 protein in mesophyll and bundle sheath chloroplast of maize. Biochim. Biophys. Acta 1787, 1161-1169. doi: 10.1016/j.bbabio.2009.05.002

Porra, R. J., Thompson, W. A., and Kriedmann, P. E. (1989). Determination of accurate extinction coefficients and simultaneous equations for assaying chlorophylls a and b with four different solvents: verifications of the concentration of chlorophyll standards by atomic absorption spectroscopy. Biochim. Biophys. Acta 975, 384-394. doi: 10.1016/S0005-2728(89) 80347-0

Puthiyaveetil, S., Tsabari, O., Lowry, T., Lenhert, S., Lewis, R. R., Reich, Z., et al. (2014). Compartmentalization of the protein repair machinery in photosynthetic membranes. Proc. Natl. Acad. Sci. U.S.A. 111, 15839-15844. doi: 10.1073 /pnas. 1413739111

Schägger, H., and von Jagow, G. (1991). Blue native electrophoresis for isolation of membrane protein complexes in enzymatically active form. Anal. Biochem. 199, 223-231. doi: 10.1016/0003-2697(91)90094-A

Shi, L. X., Hall, M., Funk, C., and Schröder, W. P. (2012). Photosystem II, a growing complex: updates on newly discovered components and low molecular mass proteins. Biochim. Biophys. Acta 1817, 13-25. doi: 10.1016/j.bbabio.2011.08.008

Suorsa, M., Regel, R. E., Paakkarinen, V., Battchikova, N., Herrmann, R. G., and Aro, E. M. (2004). Protein assembly of photosystem II and accumulation of subcomplexes in the absence of low molecular mass subunits PsbL and PsbJ. Eur. J. Biochem. 271, 96-107. doi: 10.1046/j.1432-1033.2003.03906.x

Takahashi, T., Inoue-Kashino, N., Ozawa, S., Takahashi, Y., Kashino, Y., and Satoh, K. (2009). Photosystem II complex in vivo is a monomer. J. Biol. Chem. 284, 15598-15606. doi: 10.1074/jbc.M109.000372

Tomizioli, M., Lazar, C., Brugière, S., Burger, T., Salvi, D., Gatto, L., et al. (2014). Deciphering thylakoid sub-compartments using a mass spectrometry-based approach. Mol. Cell. Proteomics 13, 2147-2167. doi: 10.1074/mcp.M114.04 0923

Watanabe, M., Iwai, M., Narikawa, R., and Ikeuchi, M. (2009). Is the photosystem II complex a monomer or a dimer? Plant Cell Physiol. 50, 1674-1680. doi: $10.1093 / \mathrm{pcp} / \mathrm{pcp} 112$

Conflict of Interest Statement: The authors declare that the research was conducted in the absence of any commercial or financial relationships that could be construed as a potential conflict of interest.

Copyright (C) 2015 Haniewicz, Floris, Farci, Kirkpatrick, Loi, Büchel, Bochtler and Piano. This is an open-access article distributed under the terms of the Creative Commons Attribution License (CC BY). The use, distribution or reproduction in other forums is permitted, provided the original author(s) or licensor are credited and that the original publication in this journal is cited, in accordance with accepted academic practice. No use, distribution or reproduction is permitted which does not comply with these terms. 